\title{
Kinetically-Driven Frustration in Hybrid Spin Ladders
}

\author{
R.C.P. Carvalho ${ }^{a}$, M.S.S. Pereira ${ }^{a}$, M.L. Lyra ${ }^{a, *}$, O. Rojas ${ }^{b}$, J StreckA ${ }^{c}$ \\ ${ }^{a}$ Instituto de Fisica, Universidade Federal de Alagoas, 57072-970 Maceio-AL, Brazil \\ ${ }^{b}$ Departamento de Ciencias Exatas, Universidade Federal de Lavras, 37200-000, Lavras-MG, Brazil \\ ${ }^{c}$ Department of Theoretical Physics and Astrophysics, Faculty of Science, P.J. Šafárik University, Park Angelinum 9, \\ 04001 Košice, Slovakia

\begin{abstract}
We introduce a spin ladder model incorporating localized and delocalized spins, which is exactly solvable using the decoration-iteration and transfer-matrix techniques. Quantum correlations between delocalized spins induce an antiferromagnetic coupling between the spin chains that competes with a direct ferromagnetic exchange coupling. A resulting kinetically-driven frustration is predominant in the regime of low temperatures and large hopping amplitudes. We provide the full ground-state phase diagram, as well as the frustration diagram on distinct coupling regimes.
\end{abstract}

DOI: $10.12693 /$ APhysPolA.126.12

PACS: 75.10.Jm, 75.10.Pq, 05.70.Ce

\section{Introduction}

Recently, a new class of hybrid spin systems was introduced with nodal localized Ising spins and interstitial delocalized electrons [1-4]. In a diamond-like geometry, this class of models displays magnetization plateaus [1] and an enhanced magnetocaloric effect [2]. In a square lattice geometry, the quantum correlations may lead to ferromagnetic [3] or antiferromagnetic [4] ordering depending on the filling fraction of the delocalized orbitals.

Here, we introduce a hybrid spin ladder model with localized and delocalized spins on which the quantum correlations between the delocalized spins induce an effective antiferromagnetic coupling between the spin chains that competes with a direct ferromagnetic coupling.

\section{Hybrid spin ladder model}

Figure 1a is a sketch of the hybrid spin ladder model we consider. The spins $S_{i}$ and $\sigma_{i}$ are located on two distinct chains, which interact with each other through a ferromagnetic exchange coupling $J_{1}$. The interaction between nearest-neighbors spins localized on the same chain is mediated by a pair of interstitial electrons that may hop between chains with hopping amplitude $t$ obeying Pauli's exclusion rule. The exchange interaction between the localized spins and interstitial electrons is denoted by $J$. If the interstitial electrons are aligned parallel, there is no mobility and the plaquette hamiltonian $H_{i}$ has a classical character. However, the four antiparallel configurations of the interstitial electrons are quantummechanically mixed due to the hopping process. An exact decoration-iteration transformation maps the present model on an affective Ising spin ladder containing Ising spins with two and four spins couplings. The effective Ising spin ladder model can be exactly solved using the standard transfer-matrix technique.

\footnotetext{
*corresponding author; e-mail: marcelo@fis.ufal.br
}

(a)

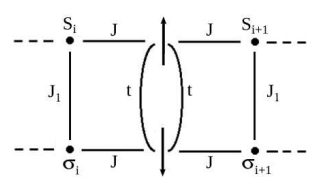

(b)

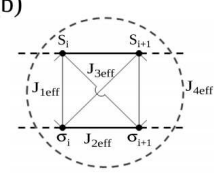

(c)

$$
\tilde{H}_{i}=\left(\begin{array}{cccccc}
|\uparrow, \uparrow\rangle & |\downarrow, \downarrow\rangle & |\uparrow, \downarrow\rangle & |\downarrow, \uparrow\rangle & |0, \downarrow\rangle & |\uparrow, 0\rangle \\
\begin{array}{c}
\mathrm{J}\left(\mathrm{S}_{1}+\mathrm{S}_{2}\right)- \\
\mathrm{J}\left(\sigma_{1}+\sigma_{2}\right)
\end{array} & 0 & 0 & 0 & 0 & 0 \\
0 & \begin{array}{c}
\mathrm{J}\left(\mathrm{S}_{1}+\mathrm{S}_{2}\right)+ \\
\mathrm{J}\left(\sigma_{1}+\sigma_{2}\right)
\end{array} & 0 & 0 & 0 & 0 \\
0 & 0 & \begin{array}{c}
-\mathrm{J}\left(\mathrm{S}_{1}+\mathrm{S}_{2}\right)+ \\
\mathrm{J}\left(\sigma_{1}+\sigma_{2}\right)
\end{array} & 0 & \mathrm{t} & \mathrm{t} \\
0 & 0 & 0 & \begin{array}{c}
\mathrm{J}\left(\mathrm{S}_{1}+\mathrm{S}_{2}\right)- \\
\mathrm{J}\left(\sigma_{1}+\sigma_{2}\right)
\end{array} & \mathrm{t} & \mathrm{t} \\
0 & 0 & \mathrm{t} & \mathrm{t} & 0 & 0 \\
0 & 0 & \mathrm{t} & \mathrm{t} & 0 & 0
\end{array}\right)
$$

Fig. 1. A diagrammatic illustration of the hybrid spin ladder model (a) and the resulting effective model (b) after the decoration-iteration transformation. (c) The Hamiltonian matrix associated with the interactions between localized and interstitial electrons $\left(H_{i}=\hat{H}_{i}-\right.$ $\left(J_{1} / 2\right)\left(S_{i} \sigma_{i}+S_{i+1} \sigma_{i+1}\right)$.

The ground-state phase diagram is shown in Fig. 2. In the absence of a direct exchange coupling between the chains $\left(J_{1}=0\right)$, the ground state consists of two ferromagnetic chains coupled antiferromagnetically. The antiferromagnetic coupling is due to the quantum correlations that develop when the interstitial electrons have distinct orientations. When the ferromagnetic exchange coupling $J_{1}$ is turned on, new ground state phases can become stable. In the regime of small hopping amplitudes $t<2 J$, a ferromagnetic phase sets up for $J_{1}>$ $\left(4 J^{2}+t^{2}\right)^{1 / 2}-2 J$. In this regime, the quantum hopping is suppressed and the interstitial spins assume the same orientation while occupying distinct sites. In the regime of strong quantum hopping $t>2 J$, the groundstate with decoupled ferromagnetic dimers predominates for $J_{1}>\left(4 J^{2}+t^{2}\right)^{1 / 2}-t$. The decoupling between the 


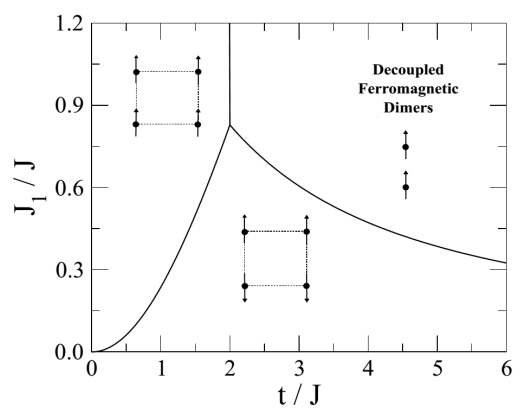

Fig. 2. Ground-state phase diagram. The decoupled ferromagnetic dimers ground-state is highly degenerated.

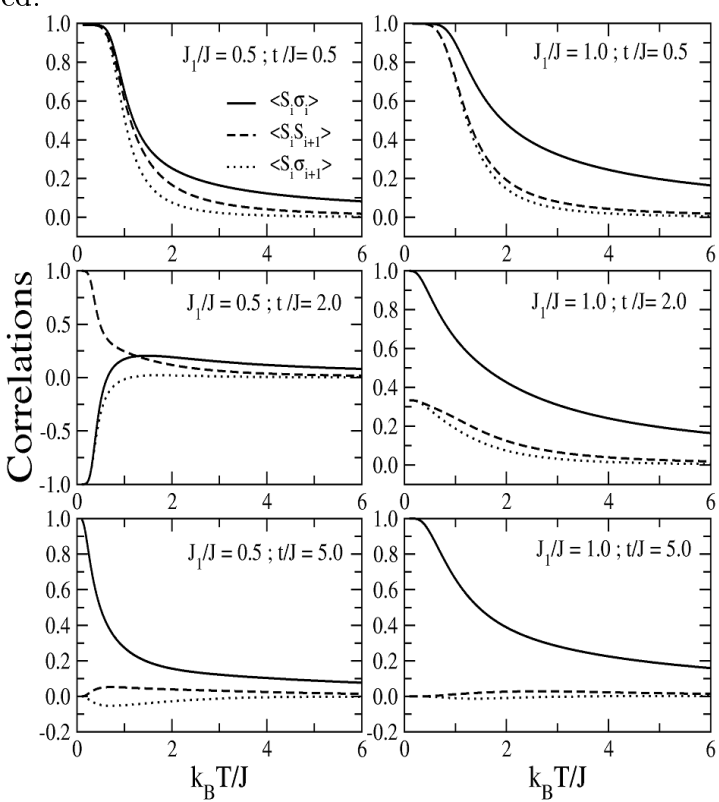

Fig. 3. Thermal dependence of pair correlation functions. Left panel: ferro, antiferro and frustrated correlations can be found for $J_{1} / J=0.5$ at low-temperature. Right panel: no antiferromagnetic correlations develop for $J_{1} / J=1.0$.

dimers is due to the strong quantum fluctuations which results from the hopping of antiparallel aligned interstitial electrons.

At finite temperatures, there is no long-range order, but the two-spin correlations within an elementary plaquette bring relevant information regarding the local predominant orientation. Figure 3 summarizes the overall scenario for two representative values of the exchange coupling $J_{1}$. For $J_{1} / J=0.5$ and small hopping $t$, the two-spin correlations around an elementary plaquette are positive, signalling the local ferromagnetic ordering. At intermediate values of $t$, correlations are consistent with a ground-state in which two ferromagnetic chains are coupled antiferromagnetically. The high-temperature correlations are ferromagnetic with an intermediate regime of frustrated correlations (the product of the three elementary two-spin correlations is negative, that indicates no predominant local ordering around the plaquette). For large values of $t$, the correlations point to a frustrated local spin configuration even at low temperatures. For $J_{1} / J=1.0$, the local antiferromagnetic order does not appear at intermediate values of $t$.

At the frustration temperature, the product of the plaquette two-spin correlations vanishes. In Figure 4, we plot the frustration diagram for the above two representative values of $J_{1}$. For $J_{1} / J=0.5$ there are three welldefined regimes. While ferromagnetic correlations predominate at high-temperatures for any strength of the hopping amplitude $t$, frustrated correlations appear as the temperature decreases when $t$ is large enough. At intermediate values of $t$, non-frustrated correlations corresponding to antiferromagnetically coupled ferromagnetic chains sets up, a regime that is absent for large values of $J_{1}$ (here illustrated by $J_{1} / J=1.0$ ).

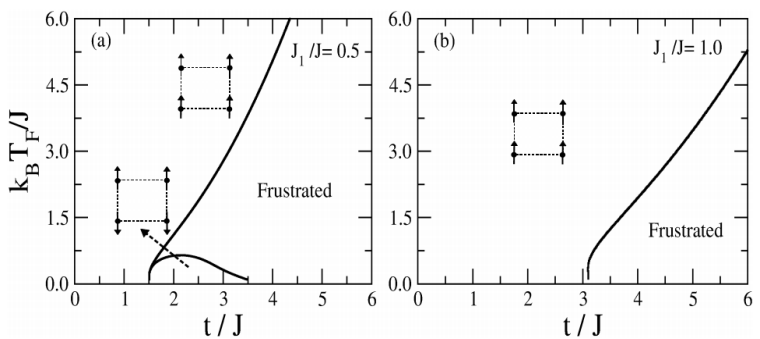

Fig. 4. Frustration diagrams. Left panel for $J_{1} / J=$ 0.5: antiferromagnetic correlations develop at low temperatures and intermediate hopping amplitudes. Right panel for $J_{1} / J=1.0$ : no antiferromagnetic correlations are developed. Frustrated correlations predominate at low temperatures and large hopping amplitudes.

\section{Conclusions}

In summary, we have introduced a hybrid spin ladder model in which a direct exchange ferromagnetic coupling between the ladder chains competes with the antiferromagnetic coupling induced by hopping process of mobile interstitial electrons. This competition leads to a new form of spin frustration that predominates at low temperatures, in contrast with the behaviour depicted by standard models of frustration induced by competitive direct exchange couplings on which frustration predominates at high temperatures. This new class of models can then be used to closely investigate the interplay between quantum and thermal fluctuations in low-dimensional spin systems.

\section{Acknowledgments}

This work was supported by the agencies CNPq, CAPES, FAPEAL, FAPEMIG, and VEGA 1/0234/12.

\section{References}

[1] M.S.S. Pereira, F.A.B.F. de Moura, M.L. Lyra, Phys. Rev. B 77, 024402 (2008).

[2] M.S.S. Pereira, F.A.B.F. de Moura, M.L. Lyra, Phys. Rev. B 79, 054427 (2009).

[3] J. Strecka, A. Tanaka, M. Jascur, J. Phys.: Conf. Series 220, 022059 (2010).

[4] J. Strecka, A. Tanaka, L. Canova, T. Verkholyak, Phys. Rev. B 80, 174410 (2010). 